\title{
Teilhabe und Öffentliche Mobilität: Die Rolle der Politik
}

\section{Stephan Daubitz}

\section{Einleitung}

Waren Verkehrspolitik und -planung in der Vergangenheit zumeist beschränkt auf die Verringerung von Raumwiderständen zur Herstellung von reibungslosen automobilen ,Verkehrsflüssen", hat sich durch die Einbeziehung von Gerechtigkeitskonzeptionen und die präzise Unterscheidung zwischen den Begriffen Verkehr und Mobilität sowie den Einbezug des Individuums mit seinen subjektiven Wahrnehmungen die Möglichkeit eröffnet, eine innovative Form von Verkehrsplanung bzw. Mobilitätspolitik zu gestalten.

Handlungsleitend ist zunehmend auch die Erkenntnis, dass Mobilität gesellschaftliche Teilhabe ermöglicht und somit ihre gerechte Ausgestaltung essentiell ist um ein ,gutes Leben“ zu führen (Sen 2012; Nussbaum et al. 2014). Inzwischen hat die Verknüpfung von Teilhabe und die Gestaltung der Mobilität auch auf kommunaler Ebene Einzug gehalten. So ist für Berlin der Stadtentwicklungsplan Verkehr und das neu geschaffene Mobilitätsgesetz handlungsleitend. In §1 des Mobilitätsgesetzes wurde „die Gewährleistung gleichwertiger Mobilitätsmöglichkeiten in allen Teilen Berlins“ als Ziel formuliert. „Damit soll für alle Personen die Teilhabe am gesellschaftlichen Leben abgesichert werden.“

Die Teilhabe hat sich zu einem Leitbegriff des wissenschaftlichen und politischen Diskurses in Hinblick auf die Zukunft des gesellschaftlichen Miteinanders entwickelt. Die zunehmende Etablierung von partizipativer Forschung und das Bemühen transdisziplinär nicht nur wissenschaftliche Ergebnisse gemeinsam zu produzieren, sondern auch Verhältnisse vor Ort zu gestalten,

S. Daubitz $(\bowtie)$

Technische Universität Berlin, Berlin, Deutschland

E-Mail: stephan.daubitz@tu-berlin.de 
ist ein Beleg dafür, dass auch in den Planungswissenschaften zunehmend das Leitkonzept der Teilhabe seine Wirkung entfaltet. Partizipative Methoden wie Community Mapping, Photo Voice, Planning for Real etc. werden in Forschungsprojekten mit Kommunen und Bürger*innen zu Fragen der Verkehrs- und Sozialpolitik angewandt (siehe den Beitrag von Hausigke \& Kruse in diesem Band).

\section{Das Leitkonzept der Teilhabe}

Materielle Ressourcen und politische Rechte bilden die wesentlichen Voraussetzungen um Teilhabe überhaupt zu ermöglichen. Dazu zählen zur Verfügung stehende Infrastrukturen ebenso wie individuelle Fähigkeiten, um Verwirklichungschancen wahrzunehmen. Die Mehrdimensionalität von Teilhabe findet ihren Ausdruck in der Identifizierung verschiedener Teilhabeformen z. B. Erwerbsarbeit, Familie bzw. soziale Nahbeziehungen (Bartelheimer 2007). So wird die Dimension der Teilhabe an Erwerbsarbeit durch die gesellschaftlichen Ressourcen der Arbeitsmarktpolitik, Unternehmen etc. bestimmt - mit dem Ergebnis der Teilhabe der existenzsichernden Erwerbsbeteiligung. Das Fehlen sozialer Ressourcen in Familie, Betrieb oder im Quartier, kann in der Dimension der sozialen Nahbeziehungen im Extremfall zur Isolation führen. Politische und soziale Rechte sowie die Teilhabe an Kultur und Bildung weisen auf die gesellschaftlichen Ressourcen des Bildungssystems, des Systems der sozialen Sicherung und den Möglichkeiten der politischen Partizipation hin.

Da Teilhabe durch individuelles Handeln in sozialen Beziehungen realisiert wird, gilt es diese individuellen Komponenten für den Bereich der Mobilitätspolitik zu berücksichtigen. In einem Begriffskanon zur Mobilitätsforschung haben die Autoren des Fachgebiets Integrierte Verkehrsplanung der TU Berlin die Begriffe Verkehr und Mobilität so definiert, dass sie für die wissenschaftliche und politische Arbeit handhabbar und praktikabel sind. Mit der Unterscheidung zwischen Verkehr (tatsächliche Ortsveränderungen) und einem erweiterten Mobilitätsbegriff, der subjektive Wahrnehmungen einbezieht, lässt sich ein Grad der Teilhabe ableiten und im Anschluss daran individuelle Möglichkeitsräume verbessern (Schwedes et al. 2018). So ist Mobilität nicht auf aktive Bewegung bzw. tatsächliche Ortsveränderungen reduziert, sondern mit der Möglichkeit verbunden seine Lebensziele zu verfolgen und umsetzen zu können, soziale Netzwerke zu bilden und aufrechtzuerhalten, sich weiterzubilden etc. Somit sind auch die Potenziale von Mobilität für ein , gutes Leben“ entscheidend. Die Möglichkeit einer Ortsveränderung befördert das Gefühl selbstständig handeln zu können und 
nicht abhängig zu sein. Um also ein ,gutes Leben` zu verwirklichen, ist Mobilität eine wichtige Fähigkeit damit Menschen ihre Bedürfnisse erfüllen können. Der Begriff des ,guten Lebens' lässt sich auch als Wohlbefinden beschreiben, der mit Vorstellungen von Glück, Lebenszufriedenheit und Erfüllung verbunden oder synonym mit diesen Begrifflichkeiten verwendet wird (Delbosc 2012; VellaBrodrick und Stanley 2013). Dementsprechend bemisst sich ein ,gutes Leben“ nicht mehr an den zurückgelegten Kilometern, sondern am Grad individueller Handlungsfähigkeit. Vor allem die angelsächsische Mobilitätsforschung hat sich den Begriff des Wohlbefindens (Well-Being) zu eigen gemacht und diesen mit der Betrachtung von sozialer Exklusion und möglichen Mobilitätseinschränkungen verbunden. Die australische Mobilitätsforscherin Alexa Delbosc (2012, S. 25) betont, dass das Hauptziel jeder Planung darin bestehe Lebensqualität und Wohlbefinden für die Bevölkerung herzustellen. Ohne die Berücksichtigung dieser Zielstellung würde Verkehrspolitik die Mobilität der Menschen nur entfremdet fördern, ohne damit negative externe Effekte eines erhöhten Verkehrsaufkommens (Lärm, Schadstoffbelastung) und die negativen physischen und psychischen Wirkungen auf das Wohlbefinden zu berücksichtigen.

Die Mobilitätsforschung, die vor allem den Ausschluss von Bevölkerungsgruppen im Blick hat und sich die Barrieren anschaut, die Mobilität erschweren und damit gesellschaftliche Teilhabe einschränken, haben einige Hinweise herausgearbeitet, auf die Mobilitätspolitik als Teilhabepolitik aufbauen kann, um politische Strategien und konkrete Maßnahmen abzuleiten. Die Forschungen geben Hinweise, welche Indikatoren zu berücksichtigen sind, um Teilhabe durch Mobilität zu ermöglichen. In der Mobilitätsforschung dominiert immer noch die verkehrsbezogene Messung, die berücksichtigt, wie leicht verschiedene Verkehrsmittel zugänglich bzw. verfügbar sind. Hier werden Barrieren erfasst, die den Zugang zu Verkehrssystemen hemmen (z. B. keine Haltestellen des ÖPNV).

Im Fokus der klassischen Erreichbarkeitsanalysen stehen zumeist die Lage von Alltagseinrichtungen. Hierbei sind große Entfernungen zu grundlegenden Einrichtungen wie Schulen, Einkaufsgelegenheiten usw. für Nutzer*innen problematisch. Finanzielle Barrieren wie beispielsweise hohe Ticketpreise für den ÖPNV verhindern, dass Einkommensarme ein Verkehrssystem nutzen können. Unter zeitliche Barrieren wird die mangelnde zeitliche Passung der Nutzer*innenbedürfnisse mit dem Verkehrsangebot (z. B. geringe Taktdichte eines Verkehrsangebots) verstanden. Des Weiteren sind die persönlichen Barrieren (physische und psychische Einschränkungen, aber auch kulturelle Barrieren) als eine Komponente der Nichtnutzung eines öffentlichen 
Verkehrssystems oder eines bestimmten Verkehrsmittels (z. B. Fahrrad) zu berïcksichtigen (Lucas 2012). ${ }^{1}$

Die Erreichbarkeitsperspektive der Mobilitätsforschung berücksichtigt bei der Untersuchung von Mobilitätsbenachteiligungen die soziale Schichtung innerhalb einer Gemeinschaft zur Identifizierung von sozialen Bedürfnissen und individuellen Nutzer*innenbedürfnissen. Solch eine Herangehensweise hatte auch immer zum Ziel, eine schlechte Versorgung zu identifizieren und zu beheben, um die mit fehlender Mobilität verbundenen Nachteile für die Betroffenen auszugleichen. Die standortbezogenen Erreichbarkeitsanalysen, Daten des Verkehrsverhaltens und des Verkehrssystems bildeten die Grundlage für diese Form der Erreichbarkeitsplanung (Accessibility Planning), die z. B. in Großbritannien etabliert wurde (Schwedes und Daubitz 2011).

Wenn es um Überlegungen z. B. zur Realisierung von Erreichbarkeitsplanungen geht oder um das Ziel mit geeigneten Maßnahmen bestimmten Bevölkerungsgruppen die Teilhabe am gesellschaftlichen Leben zu ermöglichen, bildeten im Hintergrund Vorstellungen von sozialer Gerechtigkeit zumeist den normativen Rahmen. Auch die Gründung der Social Unit in Großbritannien im Jahre 2003, die sich zum Ziel gesetzt hatte den Zusammenhang von Mobilität und Armut zu untersuchen und mit geeigneten Maßnahmen zu bearbeiten, war eingebettet in eine Debatte um soziale Gerechtigkeit. Von der damaligen Labour Regierung wurde das initiierte Programm als ein Baustein angesehen, um mehr soziale Gerechtigkeit im Lande zu realisieren. Grundlegend ist die Vorstellung, dass Ungleichheit der Lebensbedingungen (Einkommen, Bildung etc.) nicht als Ergebnis eines Wettbewerbs gesehen, sondern im Gegenteil als wichtigstes Mobilitätshemmnis bewertet wird. Dies führt zu einer funktionalen Betrachtung von Chancen bzw. Barrieren der Mobilität, die dem Ziel der Erreichung von sozialer Gerechtigkeit entgegenstehen (Hradil und Schiener 2001). Im Bereich der Gestaltung von Verkehrssystemen stellt die Herstellung und Bereitstellung von Zugängen und der Abbau von Barrieren eine zentrale zu bearbeitende Thematik dar.

\footnotetext{
${ }^{1}$ Karen Lucas hatte 2012 die internationalen Studien zu Mobilität und Exklusion gesichtet und sieben Dimensionen herausgearbeitet, die Teilhabe verhindern. Eine besondere Form der räumlichen Ausgrenzung stellen die Gated Communities dar, in denen bestimmten Gruppen der Zugang durch aktive Ausgrenzung einer sozialen Gruppe verwehrt wird. Gated Communities gibt es nicht nur in den USA oder in Lateinamerika, auch in Deutschland hat sich seit Ende der neunziger Jahre diese Wohnform verbreitet (Glasze 2002; Wehrheim 2002).
} 
Mit dem Einbezug der subjektiven Wahrnehmungen von Möglichkeitsräumen in der Mobilitätsforschung und auch in der Planung wird der eingeschränkte Blick auf die Zugänge zu Verkehrsmitteln erweitert. Somit sind beispielsweise die Kompetenzen, eine Fahrt mit dem ÖPNV zu einem fremden Ort zu planen und auch erfolgreich durchzuführen, ein ebenso wichtiger Indikator für die Realisierung von Mobilität. Fehlende Fähigkeiten, fehlendes Wissen und negative Erfahrungen bei der Nutzung von Verkehrsmitteln wirken sich negativ auf das Wohlbefinden aus.

Teilhabe im Bereich der Mobilität ist mehrdimensional und hat die Felder der Verkehrsinfrastruktur, der räumlichen Organisation, die tatsächlichen Aktivitäten, die soziokulturellen Verhältnisse und die subjektiven Wahrnehmungen im Blick (siehe Beitrag von Rammert in diesem Band).

\section{Das Konzept des Capability Approach}

Der in den Gesundheitswissenschaften und in der Armuts- und Reichtumsberichterstattung der Bundesregierung etablierte Befähigungsansatz (Capability Approach) könnte für den Bereich der Mobilitätspolitik die entscheidende normative Basis bilden (Bundesregierung 2005, S. 7). Das Konzept des Capability Approach besteht aus einer Reihe von Ideen, Konzepten und Methoden, die hauptsächlich von dem indischen Ökonomen Amartya Sen (2012) entwickelt wurden. Bei dem Konzept des Capability Approach handelt es sich um einen Ansatz, der individuelle und kollektive Wohlfahrt miteinander verbindet. Hierbei werden Armut und daraus resultierende Ungerechtigkeit nicht auf die Verteilung von Gütern reduziert.

Grundlegend für das Konzept ist es, dass die umfassende gesellschaftliche Teilhabe als anzustrebendes Maß angesehen wird. Soziale Rechte und die demokratische Verfasstheit einer Gesellschaft bilden hier die fundamentale Basis, damit Menschen Verwirklichungschancen haben. Sens Auseinandersetzung mit der Gerechtigkeitstheorie von John Rawls (1971, 2001) führte zu einer Abgrenzung zu vertragstheoretischen Gerechtigkeitstheorien. Die Entwicklung vertragstheoretischer Gerechtigkeitsprinzipien und deren Ausgestaltung setzt die Vorstellung voraus, dass keiner seinen Platz in der Gesellschaft kennt. Es ist sozusagen ein Gedankenexperiment, dass der Entwicklung und Ausgestaltung von Gerechtigkeitsprinzipien vorausgeht. Dies geschieht auf Grundlage der Vorstellung, dass noch niemand seinen Platz in der Gesellschaft gefunden hat, dass keine soziale Stellung, keine persönlichen Normvorstellungen und keine physischen und psychischen Merkmale zugeordnet wurden. Aus der Tatsache, 
dass für alle die Möglichkeit besteht, jedem Geschlecht, jeder Klasse anzugehören, würde sich also zwingend ableiten lassen, jedem/jeder Einzelnen Gerechtigkeit zu garantieren. Schließlich würde jeder Einzelne sich wünschen frei von Armut zu sein und entsprechende soziale Einrichtungen zum Schutz vor dieser Lebenssituation entwickeln. Das Ignorieren von Machtverhältnissen ist eine Hauptkritik an der Gerechtigkeitskonzeption von Rawls. In der Auseinandersetzung mit Rawls entwickelte Amartya Sen seine Idee der Gerechtigkeit. Berühmt geworden ist sein Beispiel von drei Kindern, die mit unterschiedlichen Argumenten um den Besitz einer Flöte werben. Das erste Kind argumentiert, dass es im Gegensatz zu den anderen Kindern das Instrument beherrscht. Das zweite Kind verweist darauf, dass es arm ist und sonst kein Spielzeug besitzt. Das dritte Kind führt aus, dass es die Flöte selbst hergestellt hat. Sen stellt in diesem Beispiel dar, das es durchaus eine vernünftige Pluralität gibt, die unausweichlich ist. Dies ist auch übertragbar auf die möglichen Standpunkte verschiedener Gerechtigkeitstheoretiker.

\begin{abstract}
„Wie bereits gesagt, können Theoretiker unterschiedlicher Denkrichtungen, also Utilitaristen, ökonomische Egalitarier, Arbeitsrechtstheoretiker oder nüchterne Libertäre alle der Ansicht sein, dass es eine unkomplizierte gerechte und leicht zu findende Lösung gibt, aber sie würden sich jeder für eine andere offenkundig richtige Lösung einsetzen. Es kann sein, dass es tatsächlich keine erkennbare vollkommen gerechte soziale Regelung gibt, aus der eine unparteiische Einigung hervorginge." (Sen 2012, S. 43 f.)
\end{abstract}

Da also eine Einigung über einen allgemeinen Standpunkt nicht möglich erscheint, schlägt Sen eine komparative Perspektive vor, die konkrete Ungerechtigkeiten und die Möglichkeiten diese zu bekämpfen in den Blick nimmt. Diese grundlegenden Überlegungen $\mathrm{zu}$ den individuellen Verwirklichungschancen in ihrer Abhängigkeit zu den ökonomischen, politischen und sozialen Rahmenbedingungen einer Gesellschaft beeinflusste unter anderem die Konzeptualisierung des Human Development Index der Vereinten Nationen.

Der Befähigungsansatz hat den Anspruch, die Entwicklung von Lebensqualität zu erfassen. Eine Reduktion auf Begriffe wie Glück und Zufriedenheit ist dabei für die Beurteilung von Lebensqualität nicht zielführend. Um das Konzept des Befähigungsansatzes zu verstehen, ist die Erläuterung der wesentlichen Begriffe Sens notwendig. Zentral für das Modell des Befähigungsansatzes sind die Begriffe der Funktionsweisen (functionings) und der Fähigkeiten (capabilities), die zur Verfügung stehenden Ressourcen bzw. Güter (resources), sowie die Umwandlungsfaktoren (conversion factors). Bei den Funktionsweisen (functionings) handelt es sich um die für einen Menschen tatsächlich möglichen 
materialisierten Lebenschancen, Zustände oder Tätigkeiten (Bartelheimer 2007). Fähigkeiten (capabilities) sind individuell realisierbare Funktionsweisen, also die Möglichkeit potenzielle Zustände und Tätigkeiten zu erreichen und auszuführen. Es bedarf also der individuellen Fähigkeiten sowie der Verfügbarkeit von Gütern, um Funktionsweisen realisieren zu können. Gesellschaftliche und persönliche Rahmenbedingungen (Umwandlungsfaktoren) beeinflussen die individuellen Fähigkeiten und die Verfügbarkeit von Gütern.

Der französische Soziologe Jean-Michel Bonvin (2006) hat das Konzept des Befähigungsansatzes mit seinen zentralen Begriffen an einem Mobilitätsbeispiel beschrieben und somit schon die möglichen Konkretisierungen einer Mobilitätspolitik, die sich als Teilhabepolitik begreift, aufgezeigt. Bonvin hat das Beispiel des Fahrradfahrens genutzt um zu beschreiben, wie Teilhabe ,funktionieren" kann. So wäre die Mobilität mit dem Fahrrad (resources), also die Tätigkeit des Fahrradfahrens, eine Funktionsweise (functionings). Um Fahrrad zu fahren muss die Person physisch in der Lage bzw. die Fähigkeit besitzen (capabilities), das Fahrrad zu gebrauchen. Capabilities sind also Handlungsmöglichkeiten bzw. Verwirklichungschancen, die einer Person zur Verfügung stehen. Und natürlich muss die Ressource Fahrrad überhaupt verfügbar sein.

Die Fähigkeit, bestimmte Güter in Funktionsweisen umzuwandeln, hängt von gesellschaftlichen, umweltspezifischen Umwandlungsfaktoren (conversion factors) ab. Hier können viele bestimmende Indikatoren identifiziert werden: soziale und kulturelle Normen, politische Strukturen, klimatische Bedingungen. Bezogen auf das Fahrradbeispiel wäre die Funktionsweise davon abhängig, dass es eine hinreichende Fahrradinfrastruktur gibt (Fahrradwege, Abstellmöglichkeiten etc.) oder ob es sozial oder kulturell überhaupt erlaubt ist Fahrrad zu fahren.

Tatsächlich ist es für Einkommensarme ein Problem, wenn das Fahrrad gestohlen oder beschädigt wird, da finanzielle Barrieren oft die Wiederanschaffung eines Rads verhindern. Des Weiteren muss auch eine angemessene Fahrradinfrastruktur vorhanden sein, um das Fahrrad zu nutzen. Und natürlich muss die Person überhaupt die Fähigkeit haben Radfahren zu können. Erst wenn diese Bedingungen erfüllt sind, kann von einer Wahlfreiheit gesprochen werden bzw. kann eine mögliche Mobilitätsform umgesetzt werden.

Das Beispiel verweist schon auf die möglichen Interventionen um Mobilität inklusiv zu gestalten. Zum einen ist eine angemessene Infrastruktur herzustellen, damit die Verwirklichung von Lebenszielen überhaupt gelingen kann. Zum anderen müssen persönliche Möglichkeitsräume eröffnet werden, um Teilhabe zu verbessern. 
Mobilität wird innerhalb des Befähigungsansatzes nicht als eine Fähigkeit explizit benannt. Sen hat jedoch immer den flexiblen Ansatz betont, der spezifisch schauen sollte, welche Anforderungen sich für eine freiheitsbasierte gerechte Gesellschaft stellen sollten. Dabei bezog er neben den basalen Voraussetzungen von Wohlergehen (Unversehrtheit der Person etc.) die Möglichkeit, sich Lebensziele zu setzen und diese auch zu erreichen, ausdrücklich mit ein.

Die Philosophin Martha C. Nussbaum (2000) stellte eine Kriterienliste des ,guten Lebens' zusammen, die sie aber auch nicht als statisch ansieht. Die Fähigkeit, familiäre und soziale Bindungen einzugehen, ist mit der Fähigkeit zur Mobilität verknüpft. Um familiäre und soziale Beziehungen auszubilden bzw. pflegen zu können, muss es Möglichkeiten der persönlichen Begegnung geben. Hierbei stellt die Fähigkeit mobil zu sein ein wichtiges Bindeglied dar, damit die Begegnung überhaupt möglich ist.

\section{Was verhindert eine Mobilitätspolitik als Teilhabepolitik?}

Amartya Sen (2012) und der aus Kanada stammende Gerald Cohen (2008) bezweifeln, dass liberale kapitalistische Gesellschaften Gerechtigkeitsprinzipien produzieren können. Vielmehr seien die Ungleichheiten in den sozialen Einrichtungen dieser Gesellschaften fest verankert. Nach Sen entstehen Ungleichheiten in den Situationen, in denen Rechte auf bestimmte Güter und weniger die Güter selbst fehlen. Die Ungleichheit wird durch die Beziehung des Menschen zum Gut definiert und nicht durch das Gut an sich. Die Ungleichheit ergibt sich also über den Unterschied, wer sich den Preis für die Nutzung eines öffentlichen Verkehrsmittels letztendlich leisten kann oder ihn überhaupt nutzen darf.

Einkommensarme Haushalte müssen einen viel größeren Anteil im Gesamtbudget für Mobilität aufbringen als einkommensreichere Haushalte (Altenburg et al. 2009). Die einkommensarmen Haushalte sind daher gezwungen innerhalb ihres Gesamtbudgets entsprechend umzuschichten, um Mobilität überhaupt zu realisieren. Eine weitere finanzielle Barriere ergibt sich durch die Verteuerung der Öffentlichen Mobilität. Der öffentliche Nahverkehr ist z. B. in den vergangenen Jahren in Deutschland deutlich teurer geworden. So stieg der Preis von 2000 bis 2018 um $79 \%$. In diesen 18 Jahren verteuerte sich der Kauf und der Unterhalt von Kraftfahrzeugen um $36 \%$ (destatis 2018).

Ebenso wurde mit dem Abbau des öffentlichen Personennahverkehrs vor allem im ländlichen Raum, die Abhängigkeit von einem Verkehrsmittel und deren Verkehrsinfrastruktur immer weiter manifestiert. Somit wurden Menschen der 
Möglichkeiten beraubt sich überhaupt nachhaltig zu bewegen, da die Zugänge nicht mehr oder nur noch , ausgedünnt" vorhanden sind. Somit wird auch die räumliche Komponente eine wichtige Gerechtigkeitsdimension. In Anlehnung von Sens Befähigungsansatz - also eine Abwendung in der Betrachtung von dem Besitz von Gütern hin zu den Möglichkeiten, die ein Mensch verwirklichen kann - identifizierte Jane Samuels (2005) vor allem in Megacitys Strukturen der Ungerechtigkeit. Sie präsentierte Indikatoren, die zur Messung von Freiheit dienen können. In diesem Sinne stellt das Recht auf einen urbanen Lebensraum, der die menschliche Entfaltung ermöglicht, ein wesentliches Grundgut dar. Dieses Grundgut wird durch zahlreiche Barrieren vor allem Menschen mit Einschränkungen vorenthalten. Hierzu gehören Barrieren in der Mobilität (z. B. fehlender Zugang zu Verkehrsmitteln), aber auch die Verdrängung des gesellschaftlichen Lebens von der Straße durch die autozentrierte Verkehrsplanung. Die Herstellung von Flächengerechtigkeit ist z. B. eine wesentliche zu bearbeitende räumliche Gerechtigkeitsdimension, um lebenswerte Umwelten wiederherstellen zu können. Die große verhaltensbezogene Verkehrserhebung „Mobilität in Deutschland“ zeigt, dass ein privates Kraftfahrzeug ca. $23 \mathrm{~h}$ am Tag überwiegend am Wohnort geparkt wird (Nobis und Kuhnimhof 2018, S. 5). In den Städten wird die Hälfte des öffentlichen Straßenraums von parkenden Autos besetzt, was mit hohen Kosten für die Kommunen verbunden ist. In ihrem Rechtsgutachten errechnete die Agora Verkehrswende: „So kostet die Bereitstellung eines Parkplatzes ca. 1.500 bis 5.000 Euro und die Erhaltung des Parkplatzes etwa 60 bis 300 Euro pro Jahr" (Agora Verkehrswende 2018, S. 14). Durch die Zuweisung des öffentlichen Straßenraums wird ein öffentliches Gut privatisiert bzw. der Konkurrenz ausgesetzt. Die Nutzung des Raumes ist nur einer bestimmten Gruppe möglich. Einkommensarme Menschen können sich zumeist kein Auto leisten, um ebenfalls um die Fläche zu konkurrieren.

Mit dem normativen Narrativ des Befähigungsansatzes und dem bloßen Feststellen von Gerechtigkeitslücken im Bereich der Gestaltung von Mobilität ist es jedoch noch nicht getan. Es gilt die Strukturen, die Ungerechtigkeiten entstehen lassen und diese verfestigen, $\mathrm{zu}$ benennen, $\mathrm{zu}$ beseitigen oder zu verändern. Iris Marion Young (2011) geht in ihren Überlegungen zur Gerechtigkeitstheorie den Weg über die Analyse von Ungerechtigkeit. Um zu einer angemessenen Konzeption von Gerechtigkeit zu kommen, gilt es, die Strukturen von Unterdrückung auszumachen. Dabei geht es vor allem darum auch die Machtstrukturen aufzudecken, die Ungerechtigkeit produzieren bzw. manifestieren. In ihrer Analyse geht Young von fünf Formen der Unterdrückung aus: Ausbeutung, Marginalisierung, Machtlosigkeit, Kulturimperialismus und Gewalt. Mit dieser Kategorisierung versucht Young zu zeigen, dass die Quelle von Ungerechtigkeit 
nicht allein von der Verteilung der Güter abhängt, sondern dass im Kern Unterdrückung und Ausbeutung von sozialen Gruppen an sozialen Gruppen ausgeübt wird. Eine Ursache von Ungerechtigkeit ist die fehlende Anerkennung spezifischer Lebensweisen, Kulturen und sozialer Gruppen. Dass die Differenz zwischen den sozialen Gruppen nicht anerkannt wird, ist für Young eines der Haupttreiber für Ungerechtigkeit. Somit schließt sie an anerkennungstheoretischen Modellen der Sozialphilosophie an. Als konkretes politisches Ziel lässt sich hieraus ableiten, eine grundsätzliche Anerkennung zwischen Differenzen sozialer Gruppen herzustellen.

Beispielsweise lässt sich für die Differenz von sozialen Gruppen im Bereich der Mobilität ein racial gap, also eine auf Ethnie basierende Ungerechtigkeit konstatieren. Dies schildert die Stadt-Anthropologin Adonia Lugo (2018) am Beispiel der US-Amerikanischen Fahrradkultur. Sie zeigt, wie die Debatte z. B. um die Gestaltung einer angemessenen Fahrradstruktur von einer akademischen Mittelschicht dominiert wird. Proletarisch und migrantisch geprägte Milieus sind bei den Überlegungen einer Gestaltung nachhaltiger Nahmobilität weitgehend ausgeschlossen.

Auf eine genderbasierte Differenz weisen z. B. die Mobilitätsforscher Joachim Scheiner und Christian Holz-Rau hin (2017). Frauen sind aufgrund der Kombination von Wegezwecken (trip chaining) z. B. durch Bring- und Holdienste von Kindern und Angehörigen sowie der Besorgung von Einkäufen anders unterwegs als Männer (deren Wege sich zumeist als weniger komplex darstellen). Für die Anforderungen der sozialen Gruppe der Frauen sind zum einen die räumliche Ausgestaltung des öffentlichen Nahverkehrs inadäquat, da durch die radiale Gestaltung des ÖPNV die wichtigen Unterzentren einer Stadt nicht angemessen verbunden sind. Hinzu kommt, dass es in einigen Städten und Regionen nicht möglich ist mit der gekauften Fahrkarte unkompliziert zwischen verschiedenen Verkehrsmitteln des ÖPNV zu wechseln. Dass diese Differenz nicht bedacht wird, hängt sicherlich damit zusammen, dass Verkehrsplanung und Verkehrspolitik von Männern dominiert wird. Auf eine Bundesverkehrsministerin müssen wir in Deutschland noch warten.

Neben den fehlenden und den ungleichen Zugängen zum Verkehrssystem können wir als weitere Gerechtigkeitslücke die ungleiche soziale Betroffenheit durch die externen Effekte (Luftschadstoffe, Lärm, Verkehrsunfallgefahr) von Verkehrsprozessen ausmachen (Rammler und Schwedes 2018). Im öffentlichen Diskurs um die nachhaltige Gestaltung von Mobilität wird in diesem Zusammenhang die Verbindung zur Konzeption der Umweltgerechtigkeit betont und gesucht. Die normative Konzeption der Umweltgerechtigkeit setzt sich zum Ziel, „dass alle Menschen in vergleichbarem - und möglichst geringem - Maße 
von Umweltbelastungen betroffen sind und zudem über einen gleichwertigen Zugang zu Umweltressourcen verfügen“" (Gaffron 2016). Eng verknüpft ist die Konzeption der Umweltgerechtigkeit mit dem Begriff der Gleichheit. Gerechtigkeitskonzeptionen, die sich soziale Gerechtigkeit oder Umweltgerechtigkeit zum Ziel setzen, möchten einen Zustand erreichen, in dem alle Gesellschaftsmitglieder sozial gleichgestellt sind bzw. nicht in ungleicher Weise z. B. bestimmten schädlichen Umweltemissionen ausgesetzt sind. Somit werden Ungleichverteilungen von Umweltbelastungen als ungerecht bewertet und bieten auch für Verkehrsplaner*innen konkrete Anknüpfungspunkte für die Entwicklung von Maßnahmen. Neben den Veröffentlichungen seitens der Verkehrsforschung, die sich auf die Konzeption der Umweltgerechtigkeit beziehen und der Etablierung des Fachs Umweltgerechtigkeit im Rahmen eines Studiums des Verkehrswesens an der TU Berlin dienen, zeigt sich das Bemühen sozial-räumliche Verteilungen von verkehrsbedingtem Lärm und Luftschadstoffen $\mathrm{zu}$ beschreiben bzw. zu bewerten (Becker 2016).

Während das Konzept der Umweltgerechtigkeit auf die gegenwärtig beobachtbaren Umweltbelastungen fokussiert ist, bezieht das Konzept der Nachhaltigkeit die inter- und intragenerationale Gerechtigkeit ein (World Commission on Environment and Development 1987). Der vorgestellte Befähigungsansatz kann sich zum einem mit dem zugrunde liegenden Menschenbild auf das Ziel der nachhaltigen Entwicklung beziehen, denn die Protagonist*innen des Befähigungsansatzes halten den Menschen für fähig sich für Werte und Interessen anderer Menschen bzw. Generationen einzusetzen (Max-Neef 1991; Nussbaum 2000). Zum anderen ist die Nachhaltigkeitskonzeption auf die Bedürfnisse der Menschen auch kommender Generationen ausgerichtet. Dies ist durchaus kompatibel zu dem Begriff der Verwirklichungschancen und den damit verbundenen Freiheiten (Alkire 2005; Rauschmayer et al. 2011).

\section{Vision der Öffentlichen Mobilität}

Öffentliche Mobilität wird oft mit der Nutzung von öffentlichen Verkehrsmitteln gleichgesetzt. Dass selbst die öffentlichen Verkehrsmittel als nicht öffentlich wahrgenommen werden, zeigt folgendes Interviewzitat: „Ich störe mich auch an der Bezeichnung öffentliches Verkehrsmittel, man wird nebenbei kriminalisiert, wenn man ohne Ticket unterwegs ist", so reagiert ein 40-Jähriger Hartz-IVEmpfänger aus Hamburg auf das Thema öffentlicher Nahverkehr in Hamburg. In einem Interview, das im Rahmen des von der Deutschen Forschungsgemeinschaft (DFG) finanzierten Forschungsprojekts „,mobileinclusion“ entstanden ist, führt er 
weiter aus: „Du musst nur ein Fahrrad kaufen. Das ist ein öffentliches Verkehrsmittel, das jeder nutzen kann. Jeder kann damit fahren. Ja, würde ich sagen. Also es gibt keine Gebühren für Fahrräder.“

Diese Einschätzung macht erst einmal stutzig, da man U-Bahn, Bus, Straßenbahn als öffentlich versteht und das Fahrrad als ein privates Verkehrsmittel bezeichnen würde. Aber die interviewte Person thematisiert Erfahrungen der Kriminalisierung, die sie durch ,Schwarzfahren' erlebt hat und benennt die generelle finanzielle Barriere, die ihm die Nutzung des öffentlichen Nahverkehrs verwehrt. Für Andere, die von Hartz IV, Minijobs etc. leben müssen, ist auch der Zugang zum Fahrrad bisweilen nicht möglich, da hierfür das Geld fehlt sich ein neues Fahrrad zu kaufen bzw. ein beschädigtes Rad mit entsprechenden Ersatzteilen zu reparieren. Jedenfalls sind es wohl vor allem finanzielle Zugangsbarrieren, die Öffentliche Mobilität als nicht öffentlich erscheinen lassen. Die benannten bzw. beobachteten Barrieren zeigen ungerechte Nutzungschancen bzw. ungleiche Zugänge zu Verkehrssystemen auf.

Der vorgestellte Befähigungsansatz begreift Freiheit als eine, die neben der Abwesenheit von Zugangsbarrieren die Anwesenheit von realen Möglichkeiten voraussetzt. Die Strukturen einer Gesellschaft, die vom Automobil abhängen, schränken die Wahlmöglichkeiten und Verwirklichungschancen ein. Diejenigen, die es nicht schaffen mit dem Automobil große Pendlerdistanzen zu bewältigen, können nicht arbeiten oder haben weniger Chancen einen angemessenen Job zu bekommen. Geringfügig Mehrfachbeschäftigte sind mit zwei, drei Jobs gezwungen viel für ihre Mobilität auszugeben, ohne sich gesellschaftliche Teilhabe leisten zu können. Folgt man dem normativen Leitbild der Teilhabe und dem Befähigungsansatz, ist es evident die Mobilität der Menschen und das Verkehrssystem neu zu organisieren. Die Freiheit von Zwängen muss zwangsläufig mit der Bereitstellung von Möglichkeiten zur Erreichung von Lebenszielen gekoppelt sein.

Eine Vision von Öffentlicher Mobilität könnte eine von Zugangsbarrieren und Eigentumsansprüchen freie Organisation von Mobilität sein. Sowie eine Mobilität, die Menschen nicht nur wieder zueinander bringt bzw. sozial näher rücken lässt, sondern die von den Bürger*innen selbst gestaltet wird. Die Entwicklung und Planung von Verkehrsprojekten wird nicht nur Verkehrsplaner*innen überlassen, sondern von den Bürger*innen mit ihren Kompetenzen sowie ihrem Praxiswissen mitbestimmt. Den Verkehrsplaner*innen fällt also bei der Verwirklichung dieser Vision eine zunehmend moderierende Rolle zu, deren Aufgabe es ist, technische Informationen verständlich $\mathrm{zu}$ vermitteln und den Planungs- und Umsetzungsprozess transparent zu gestalten (hierzu gehört eine 
Dokumentation, aus der sich die getroffenen Entscheidungen auch nachvollziehen lassen). Durch das Nachdenken über die Gestaltung der eigenen Mobilität und die Organisation des Verkehrs im eigenen Wohnumfeld, wird Mobilität öffentlich verhandelt. Dies geht über die Umgestaltung des planerischen und kommunalpolitischen Handelns hin $\mathrm{zu}$ intersektoralen, interdisziplinären und transdisziplinären Arbeits- und Planungsstrukturen hinaus (Schwedes und Rammert 2020). Vielmehr soll eine umfassende Partizipation für alle Bevölkerungsgruppen ermöglicht werden. Am Ende könnte die selbstständige Organisation eines Quartiers und der Mobilität ihrer Bewohner*innen stehen, indem sie ihr Quartier und ihre Mobilität als Gemeingut begreifen und organisieren (Brocchi 2017).

Michael Wright et al. (2007) haben in einer Stufenleiter die Partizipation in Nicht-Partizipation, Vorstufen der Partizipation und Partizipation unterschieden. Ihre Stufenleiter entwickelte sich aus dem Kontext der Gesundheitsförderung. Partizipation bedeutet für die Autor*innen ,[...] nicht nur Teilnahme, sondern auch Teilhabe an Entscheidungsprozessen (Entscheidungsmacht). Je mehr Einfluss jemand auf einen Entscheidungsprozess nimmt, umso größer ist seine bzw. ihre Partizipation“ (Wright et al. 2009). Die Autor*innen haben damit die Arbeit von Sherry R. Arnstein aus dem Jahre 1969 aufgenommen und weiterentwickelt, die in ihrer Kritik an den Ritualen der ,Scheinpartizipation“ im Rahmen von Stadtentwicklungsprozessen eine Unterscheidung verschiedener Partizipationsstufen für nötig hielt.

Bürger*innen Informationen zu geben, nach ihrer Meinung zu fragen und vielleicht noch ihre Lebensweltexpertise einzuholen und diese in die Überlegungen von Planungen zu berücksichtigen, sind nur Vorstufen der Partizipation. Es macht durchaus Hoffnung, dass qualitative Interviews oder Methoden wie z. B. das Community Mapping von Verkehrsplaner*innen genutzt werden, da die Teilnehmer*innen Kritik üben und Ideen einbringen können. Eine Garantie auf die Berücksichtigung der Ideen haben die Teilnehmer*innen jedoch nicht. Das Ziel sollte sein, Entscheidungskompetenzen oder gar Entscheidungsmacht abzugeben.

Scheint für politische Entscheidungsträger*innen oder Mitarbeiter*innen der Verwaltung Entscheidungsmacht abzugeben schon undenkbar, ist auch die Abgabe von Kompetenzen an Bürger*innen, organisatorische, inhaltliche Entscheidungen eigenständig zu treffen, nicht einfach, da Regelwerke, Verordnungen und das Planungsrecht dem entgegenstehen. Da sich diese Regelwerke etc. nicht schnell transformieren lassen, kann die Etablierung von informellen Planungsinstrumenten eine sinnvolle Ergänzung sein. 
Werden Freiräume der Selbstverantwortung nicht gewährt oder eingeschränkt, erlahmt das Engagement und zuvor aktive Personen ziehen sich zurück. Im Rahmen des vom Bundesministerium für Verkehr und digitale Infrastruktur geförderten partizipativen Forschungsprojektes ,2Rad-1Kauf-0Emission - Radverkehr als Perspektive für den innerstädtischen Einzelhandel“" mussten Einzelhändler*innen der Schönhauser Allee in Berlin-Pankow ähnlich frustrierende Erfahrungen machen. Einige Händler*innen äußerten den Willen, die Baumscheiben vor ihren Ladengeschäften durch Bepflanzungen und Umrandungen mit kleinen Sitzflächen aufzuwerten - sofern sie dies nicht ohnehin schon taten. Jedoch gestaltete sich dies nicht so einfach, da die Bepflanzung von Baumscheiben aus Sicht des Bezirks mit diversen Konflikten (z. B. Gewährleistung der Verkehrssicherheit) behaftet ist. Noch während der Projektlaufzeit wurde dies deutlich, als die von einer Händlerin angelegte (und mit einem Blumenhändler auf die Bedürfnisse des Baums abgestimmte) Bepflanzung einer Baumscheibe von Mitarbeiter*innen des Straßen- und Grünflächenamts ohne Absprache restlos entfernt wurde. Daraufhin herrschte spürbare Unsicherheit darüber, was machbar ist und was nicht, woraufhin die Motivation der Händler*innen, sich hier möglicherweise weitergehend zu engagieren, letztlich abbrach.

In dem Moment, in dem Menschen anfangen sich den öffentlichen Raum anzueignen, setzen neue Aushandlungsprozesse ein, die möglichst in der direkten persönlichen Kommunikation zu klären sind. Einerseits ist die Offenheit für das Experimentieren als politische Kultur eine Grundvoraussetzung, um Freiräume zu eröffnen, andererseits müssen Verbindlichkeiten bzw. Regeln festgelegt werden, die das Engagement erst verstetigen. Dies muss von allen Akteuren, die sich für ihr Wohnumfeld oder die Neugestaltung von Mobilität engagieren, gemeinsam entwickelt und beschlossen werden. Dafür gibt es mittlerweile eine Vielzahl von kooperativen Planungsformaten (z. B. Planungswerkstätten, Runde Tische, etc.).

\section{$5 \quad$ Strategien einer teilhabeorientierten Mobilitätsplanung}

Eine Verbindung zwischen der Entwicklung von den Ideen der Bürger*innen bis zu deren Realisierung können von der Kommune eingestellte und finanzierte Mobilitätsbeauftragte herstellen. Im Rahmen der Vision Mobilität öffentlich und transparent $\mathrm{zu}$ gestalten, stellen diese neu geschaffenen Stellen eine wichtige Kontaktstelle zwischen professionell-institutioneller Perspektive und den Bürger*innen vor Ort dar. In einigen Kommunen werden diese Stellen zunehmend eingerichtet und finanziert. Jedoch sollten die zukünftigen 
Mobilitätsbeauftragten in ihren Aufgabenstellungen nicht nur darauf reduziert werden umweltfreundliche Verkehrsprojekte mit der Expertise als Verkehrsplaner*in umzusetzen. Mobilitätsbeauftragte sollen in erster Linie Teilhabe im Bereich von potenzieller Mobilität und realisiertem Verkehr ermöglichen und organisieren. Also konkret: Mobilitätsbeauftragte sind im Rahmen dieser Vision aufsuchend, proaktiv, persönlich unterwegs und versuchen auch schwer erreichbare Bevölkerungsgruppen für den Bereich Mobilität zu aktivieren. Dies kann ein*e Mobilitätsbeauftragte*r nicht allein, sondern sollte (wo vorhanden) auf eine funktionierende soziale Infrastruktur zurückgreifen. Die professionellen Sozialarbeiter*innen, Sozialberater*innen, Erzieher*innen, Quartiersmanager*innen etc., die in Nachbarschaftsheimen, sozialen Trägerschaften, Kirchengemeinden sowie Vereinen arbeiten, bilden eine wichtige Schnittstelle, um diese Verbindung zu der professionell-institutionellen planerischen Perspektive herzustellen. Praktisch geht es darum die Bedarfe der Bevölkerung, mit den Planungen und Fachabteilungen zu managen.

Ziel sollte es also sein, die sozialstrukturell unausgewogene Beteiligung (Meunier 2006) im Bereich der Mobilitätspolitik zu beseitigen. Mit Migrant*innen, Einkommensarmen, Kindern und Jugendlichen, Alleinerziehenden, körperlich oder psychisch eingeschränkten Personen wie auch älteren Menschen gilt es gemeinsam Formate zu entwickeln, um die Dominanz von zumeist akademisch gut ausgebildeten männlichen Bürgern zurückzudrängen. Dies ist nicht einfach und geht sicherlich auch nicht von heute auf morgen. Denn, um überhaupt angemessene lebensweltorientierte Mobilitätskonzepte zu realisieren, müssen die schwer erreichbaren Bevölkerungsgruppen erst einmal in die Lage versetzt werden Ursachen für mobilitätsbezogene Probleme in ihrem Alltag zu benennen und für sich Lösungsstrategien zu entwickeln. Hier setzen Strategien, Maßnahmen und Methoden an, die Kompetenzen und Fähigkeiten individuell entwickeln.

Die Eröffnung von persönlichen Möglichkeitsräumen mit dem Befähigungsansatz hat sich in der sozialen Arbeit bewährt und viele Projekte entstehen lassen, die bei der Entwicklung von individuellen Fähigkeiten ansetzen. Die Methoden der sozialen Arbeit wie Sozialberatung (Weitergabe von Informationen), Kompetenztraining sowie Netzwerkarbeit werden inzwischen auch auf spezifische Projekte übertragen, deren Hauptziel es ist Mobilitätskompetenzen zu erweitern.

Ein Projekt, das Migrantinnen befähigt ein Fahrrad zu nutzen, bietet z. B. das Projekt \#BIKEGEES ${ }^{2}$ an. Der Berliner Verein \#BIKEYGEES e. V. hilft

${ }^{2}$ https://bikeygees.org/ 
Migrantinnen durch Radfahrtrainings mobiler zu werden - und schafft damit die Voraussetzung für Teilhabe am gesellschaftlichen und kulturellen Leben. Mittlerweile ist der Verein Mitglied des Paritätischen Wohlfahrtsverbands und hat sich in Berlin als eine Anlaufstelle für Frauen etabliert. Frauen und Mädchen mit und ohne Fluchterfahrung können an kostenlosen Radfahrtrainings teilnehmen, wobei neben den praktischen Fähigkeiten des Fahrradfahrens auch theoretischer Verkehrsunterricht in verschiedenen Sprachen angeboten wird. Zudem lernen die Frauen ihr Fahrrad selbst instand zu halten. Viele der ehemaligen Teilnehmerinnen fungieren als Multiplikatorinnen: Sie führen selbstständig Trainings durch und geben ihr Wissen direkt weiter.

In Köln wurde ebenfalls der Ansatz der Peer-Education genutzt, um Senior*innen für die Nutzung des ÖPNV zu gewinnen. Ausgewählte Abonnent*innen des Aktiv60Tickets der Kölner Verkehrsbetriebe (KVB)

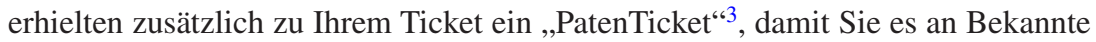
und Verwandte in ihrer Altersgruppe (die bislang nicht oder selten mit Bus und Bahn unterwegs sind) verschenkten. In einer Nachbefragung bei den Teilnehmer*innen der Aktion gaben $58 \%$ an den ÖPNV nun häufiger nutzen zu wollen und $48 \%$ der Befragten nutzten auch tatsächlich den ÖPNV. Ein weiteres messbares Ergebnis bei den Teilnehmer*innen war der Kompetenzgewinn bei der ÖPNV-Nutzung (z. B. Fahrpläne lesen können, Verbesserung der Orientierung beim Umstieg, Fahrscheinkauf etc.). Der Kompetenzgewinn war vor allem bei der Gruppe der regelmäßigen Autonutzenden signifikant.

Nun ist capacity building nicht nur auf der individuellen Ebene gefragt bzw. wichtig, sondern ebenso müssen vor Ort Strukturen aufgebaut werden, die es ermöglichen sich kontinuierlich auszutauschen, Kompetenzen zu entwickeln etc. Diese Strukturen können recht unterschiedlich aussehen bzw. sich herausbilden.

Eine vielversprechende Möglichkeit ist das Format der Reallabore (Wanner und Stelzer 2019), das sich langsam in der Forschungslandschaft etabliert. In einem Reallabor sollen zukunftsfähige, soziale, nachhaltige Strukturen und Lebensformen erprobt werden. Interessant ist der Ansatz, da im Idealfall verschiedene Akteure (Politik, Verwaltung, Wissenschaft, Zivilgesellschaft) mit ihren unterschiedlichen Kulturen aufeinandertreffen und gezwungen sind gemeinsame Strukturen zu schaffen. Im Zusammenhang mit der Förderung solcher Reallabore entstehen zumeist interessante Maßnahmen, die von den Bürger*innen stärker

\footnotetext{
${ }^{3}$ https://www.mobilservice.ch/admin/data/files/news_section_file/file/2777/koelner-projektpatenticket.pdf? $1 \mathrm{~m}=1418801210$
} 
angenommen bzw. selbst betrieben werden. Aber wie nachhaltig sind diese Strukturen, wenn die Finanzierung ausläuft? Können dann die entwickelten Maßnahmen aufrechterhalten bleiben? Beispielsweise ist bei den Fahrradwerkstätten für Einkommensarme, die meist unter sozialer Trägerschaft betrieben werden, eine permanente Fluktuation zu beobachten. Sobald die Förderung wegfällt, ist auch das Angebot und die dazugehörigen Ansprechpartner*innen hinfällig (obwohl das Angebot in den Wohnquartieren gut angenommen wurde).

Eine nachhaltige Struktur solcher Maßnahmen kann nur geschaffen werden, wenn eine Finanzierung weiterhin garantiert werden kann und/oder sich stabile Selbstverwaltungsstrukturen herausgebildet haben. Bei räumlichen Veränderungen ist die Erwartungshaltung auf stabile Strukturen schon höher, da räumliche Tatsachen geschaffen werden, die nur mit einem gewissen Aufwand wieder abgebaut werden können.

Die Idee der Superblocks, die im Barcelona realisiert wird, ist z. B. ein Konzept Stadtquartiere autofrei zu gestalten. Das Konzept der Superblocks ist ein Beispiel, um durch räumliche Absperrungen von Durchfahrten in Wohnquartiere für Automobile und die auf Fußgänger*innen und Radfahrer*innen ausgerichtete Gestaltung des abgesperrten Raumes soziale Prozesse zu ermöglichen, die sich über den Ablauf von Projektlaufzeiten erhalten können. Das im Stadtteil Poblenou in Barcelona erprobte Konzept des Superblocks soll nun auch in Berlin ausprobiert werden. Das Modell des Superblocks zu übertragen ist mit der Erwartung verbunden, dass mit der Nutzung des nun wirklich öffentlich zur Verfügung stehenden Raums mehr soziale Interaktion stattfindet und damit neue Ideen des Zusammenlebens entstehen. Aus den Erfahrungen aus Barcelona lassen sich schon positive Schlussfolgerungen ziehen (López et al. 2020). Die Superblocks in Barcelona wurden von den Anwohner*innen selbst in einem partizipativen Prozess mit der Stadtverwaltung gestaltet, um die Probleme oder Herausforderungen gemeinsam zu definieren und gemeinsam Lösungen zu finden. In jedem Distrikt, in dem das Programm durchgeführt wurde, wurde eine Lenkungsgruppe eingerichtet, die aus einer Gruppe von Personen und/ oder Vertretungsorganen bestand, um das Projekt zu überwachen. Die Gruppe fungierte als Bindeglied zwischen der professionell-institutionellen Seite und den Bewohner*innen, validierte die verschiedenen Phasen der Umsetzung sowie der Ergebnisse der Beteiligungsworkshops und der durchzuführenden technischen Maßnahmen. Die aktive und vielfältige Zivilgesellschaft in Barcelona war ein Schlüsselakteur bei der Umsetzung der Superblocks in der Stadt. Die Akzeptanz der Bewohner*innen der Superblocks ist weiterhin sehr hoch und für Kommunalpolitiker*innen könnte es eine nicht unwesentliche Information sein, dass die 
Stadtregierung Barcelonas (die das Projekt gefördert hat) wiedergewählt worden ist.

Bei dem Vorhaben, Mobilitätspolitik als Teilhabepolitik zu begreifen und diese umzusetzen, geht es auch darum sich einen kooperativen Lebensstil anzueignen bzw. diesen zu etablieren. Deshalb genügt die Kenntnis von Regelwerken für Verkehrsplaner*innen, die Mobilität öffentlich und teilhabend gestalten möchten, nicht mehr. Sondern zum zukünftigen ,Werkzeugkasten “ auf der professionellinstitutionellen Seite gehört Offenheit, Flexibilität sowie kommunikative Kompetenzen (z. B. Fähigkeiten in Moderationstechniken). Das Manipulieren, Verstecken, Verzerren von Informationen, das Ablenken der Aufmerksamkeit von wichtigen Fragen und die Verlagerung der Debatte durch Ausübung von Macht bzw. die Betonung des Anspruchs auf Fachwissen, lassen hingegen eine Kommunikation auf Augenhöhe nicht zu Stande kommen (siehe Beitrag von Schneidemesser in diesem Band). Die Arbeiten „Communicative Turn in Planning“ von Patsy Healey $(1996,2012)$ und „Argumentative Turn in Public Policy" von Frank Fischer und Herbert Gottweis (2012) bieten gute Hinweise, wie Kommunikation erfolgreich gestaltet werden kann. Die Basis ist sicherlich eine gemeinsame kommunikative Ebene zu finden und sich nicht, wie bei einberufenen Bürgerversammlungen die Partizipation suggerieren, gegenseitig zu beschimpfen.

Die Fähigkeit zur Offenheit bedeutet, dass man sich nicht hinter Regelwerken versteckt, sondern neue Ideen aus der Zivilgesellschaft abwägt und, wenn diese erfolgversprechend sind, auch umsetzt. Offenheit bedeutet auch bereit zu sein, neue Methoden auszuprobieren oder Methoden gar zu wechseln (wenn diese nicht zielführend sind). Verbal-Argumentative Methoden (z. B. SWOT-Analysen) der Dokumentation und Strategieentwicklung, die qualitative Daten (Interviews, Bild- und Filmanalysen, Beobachtungen etc.) einbeziehen, werden dabei immer wichtiger (Rammert et al. 2019).

Kontrolle und Verantwortung abzugeben fällt oft schwer, ist aber notwendig, wenn Teilhabe gelingen soll. Deshalb gehört die Fähigkeit des Vertrauens sicherlich auch in den ,Werkzeugkasten “ von Planer*innen und politischen Entscheidungsträger*innen.

Bei dieser vorgestellten Vision von Öffentlicher Mobilität wird eine Strategie von „unten nach oben“ (bottom up) präferiert. Nur hier können lokal neue Raumstrukturen geschaffen werden, die Nahmobilität ermöglichen. Das Wohnumfeld gemeinsam mit seinen Bewohner*innen in der Aufenthaltsqualität, den Möglichkeiten der Nahversorgung und der Naherholung aufzuwerten, bringt die unterschiedlichsten Gruppen der Gesellschaft wieder zusammen. Die Wahrnehmung bzw. Erfahrung selbstwirksam bei der Verwirklichung von Zielen zu sein stärkt 
zivilgesellschaftliche lokale Strukturen und macht eine Gesellschaft gegenüber undemokratischen und neoliberalen Lösungsmodellen immuner.

Trotz alledem: Angesichts der Notwendigkeit, möglichst schnell eine Mobilitätswende in Anbetracht des Klimawandels umsetzen zu müssen, sind nun auch Strategien „,von oben nach unten“ (top down) gefragt, um zum Ziel zu kommen. Dass eine Synthese zwischen beiden Strategien gelingen kann hat z. B. die Umsetzung des Nichtraucherschutzgesetzes im Rahmen der Gesundheitsprävention bewiesen, die einiges verändert hat: So sind z. B. rauchfreie Restaurants völlig selbstverständlich geworden und viele Menschen wundern sich heute darüber, wie man es in vollgerauchten Restaurants und Gaststätten überhaupt ausgehalten hat. Teilhabe im Bereich des Feldes der Verkehrspolitik zu ermöglichen heißt auch bestehenden behindernden und verhindernden Machtstrukturen zu begegnen und diese zu ersetzen. Das Herstellungs- und Kaufverbot von Verbrennungsmotoren, der Abbau von Entfernungspauschalen etc. sind notwendige Maßnahmen um die Veränderungen im Bereich der Mobilität voranzutreiben.

Parallel zu diesen restriktiven Maßnahmen sind die Zugangsbarrieren zu umweltfreundlichen Verkehrsmitteln zu beseitigen. Hierzu gehören vor allem soziale Preise für die Nutzung der Bahn und des ÖPNV, der Ausbau vor allem der Bahn- und Businfrastruktur in der Fläche und eine optimale integrierte Abstimmung der Netze (hohe Taktdichten), der Ausbau einer sicheren Fuß- und Radverkehrsinfrastruktur, um nur einige Maßnahmen zu benennen.

\section{Fazit}

Zunehmend setzt sich der Gedanke durch, dass die Gestaltung von Mobilität mit Gerechtigkeitsfragen verbunden ist. Somit wird Mobilitätspolitik automatisch zu einem normativen Vorhaben, das idealerweise zum Ziel hat Möglichkeitsräume beziehungsweise Verwirklichungschancen von Bürger*innen zu gestalten. Die Teilhabe wird zum zentralen Gradmesser für das Gelingen inklusiver Mobilitätspolitik. Voraussetzungen dafür, ein ,gutes Leben ' $\mathrm{zu}$ führen, ist die Förderung von umweltgerechter, nachhaltiger Mobilität. Mit den Folgen gesundheits- und umweltschädlicher Emissionen wird die individuelle Selbstverwirklichung erschwert. Um dies zu vermeiden müssen grundsätzliche Entscheidungen getroffen bzw. Aufgaben erfüllt werden. Zum einen muss sich davon verabschiedet werden, dass Mobilität über den Markt geregelt werden sollte. Mobilität ist eine Aufgabe der Daseinsvorsorge. Zum anderen geht es aber auch um die Selbstermächtigung der Bürger*innen sich miteinander über eine verantwortungsvolle Mobilität zu verständigen und darüber zu entscheiden. Es sind vor allem soziale Innovationen, die für den Erfolg inklusiver Mobilitätspolitik 
bestimmend sind. Hier werden zunehmend bestehende Orte der sozialen Infrastruktur wichtiger (z. B. Nachbarschaftsheime, Fahrradwerkstätten unter sozialer Trägerschaft, Lebensmitteltafeln, aufsuchende Sozialarbeit etc.). Überall, wo sich Menschen in Quartieren oder in ihrem Wohnumfeld begegnen, sollte, kann und muss die Transformation von Mobilität zum Thema gemacht werden.

Selbstermächtigung steht also als Vision für inklusive Mobilitätspolitik im Vordergrund. Mit ,Real-Experimenten“ oder ,Reallaboren` können erste Gehversuche unternommen bzw. erste Strukturen geschaffen werden, um verschiedene Perspektiven und Akteure dauerhaft zusammenzubringen. Der Befähigungsansatz von Sen und Nussbaum kann eine verbindende Grundlage für die Mobilitätspolitik zu anderen Politikfeldern sein. In der Sozialarbeit und in der Gesundheitspolitik hat sich der Befähigungsansatz etabliert. Aber auch noch dauerhaft zu etablierende neue Verkehrsplanungsinstrumente, wie z. B. kommunale Mobilitätsberichterstattungen, die partizipativ die Mobilität erheben und mit den handelnden Akteur*innen vor Ort Strategien und Maßnahmen entwickeln, können solche Strukturen inklusiver Mobilitätspolitik abbilden. Ein zu entwickelnder Mobilitätsindex, der die Möglichkeitsräume vor Ort transparent abbildet, könnte Bürger*innen z. B. selbstermächtigen sich Informationen über ihre Mobilitätsmöglichkeiten im Vergleich zu anderen Wohnorten zu besorgen.

Selbstermächtigung bzw. Selbstbefähigung steht und stand auch bei allen Projekten und Maßnahmen im Vordergrund, die Mobilität gezielt auf einzelne Personen bezogen haben und für sie ermöglichen sollen. Peer-Education Konzepte wie Fahrradlernkurse für Migrant*innen von Migrant*innen oder die Patentickets der Kölner Verkehrsbetriebe für Senior*innen von Senior*innen haben sich als erfolgreiche Einzelprojekte profiliert. Diese Projekte gilt es dauerhaft zu etablieren und die Fluktuation aufgrund fehlender Finanzierung zu vermeiden. Die Evaluierung der umgesetzten Projekte sollte zum Standard gehören. Diese Projekte der individuellen Selbstbefähigung sind notwendig, jedoch sollte man sich immer über deren Reichweite im Klaren sein. Diese Projekte können inklusiver Mobilitätspolitik nicht allein zum Erfolg helfen, sondern sind nur ein wichtiger Baustein für eine generelle politische Strategie Mobilität inklusiv zu gestalten.

Letztendlich muss eine inklusive Mobilitätspolitik die Rechte auf Räume, Zugänge und Güter im Bereich des Politikfeldes thematisieren und bearbeiten. Das sind grundlegende systemische Entscheidungen, die aber aufgrund des Klimawandels und der Umweltbelastungen durch den Verkehr unumgänglich sind. Hier reicht eine bottom-up-Strategie leider nicht aus, da es hierfür sehr viel Zeit braucht, um diese nachhaltig zu etablieren. Die Zeit drängt Nahmobilität zu ermöglichen, in der eine Öffentliche Mobilität in ländlichen und urbanen 
Räumen zu sozialen Preisen zur Verfügung steht. Politik wird oft mit dem Bild des Bohrens von dicken Brettern operiert, um metaphorisch den mühseligen zeitraubenden Prozess zu umschreiben. In der Mobilitätspolitik ist eher ein Bohren bzw. Aufbohren von dickem Beton vonnöten. Dies ist sicherlich das Dilemma, vor dem engagierte Menschen in der zu gestaltenden Mobilitätspolitik stehen, da die zu gestaltende Mobilitätswende zu spät bzw. viel zu langsam kommt, um Klimawandel und grundlegende Umweltschädigungen noch reversibel zu gestalten.

Die Umsetzung inklusiver Mobilitätspolitik läuft also keineswegs konfliktfrei ab, da das Ringen um ein ideales Verkehrssystem stark von sehr unterschiedlichen Mobilitätsvorstellungen (automobile Kultur versus postfossile Mobilitätskultur) innerhalb einer Gesellschaft geprägt wird (siehe Beitrag von Hoor in diesem Band). Begleitend zu einer konsequenten top-down-Strategie für eine inklusive und ökologische Mobilitätspolitik müssen Foren und Räume installiert werden, in denen sich unterschiedliche Mobilitätsmilieus begegnen. Es gilt die ungleichen Ressourcen, Interessen und Mentalitäten der Akteure sichtbar zu machen, um Moralisierungen zu vermeiden und einen Diskurs über die divergierenden Vorstellungen zur Mobilitätsgerechtigkeit führen zu können. Im Zusammenspiel einer top-down-Strategie und der Entwicklung disperser lokaler sozialer Strukturen, die Menschen miteinander befähigen verantwortlich ihre Mobilität zu organisieren, kann inklusive Mobilitätspolitik dauerhaft bestehen.

Mit dem Beispiel des Fahrradfahrens von Jean-Michel Bonvin haben wir gesehen, dass die Fülle der Verwirklichungschancen im Bereich der Mobilität von der tatsächlichen Erreichbarkeit bzw. Verfügbarkeit abhängt, den persönlichen Umwandlungsfaktoren (also Fähigkeiten Mobilitätsdienste zu nutzen) und den gesellschaftlichen Umwandlungsfaktoren (der Konstitution des Verkehrssystems). So ist eine dominante automobile Mobilitätskultur ein bestimmender Faktor dafür, dass Öffentliche Mobilität als nicht akzeptabel erscheint. Im Sinne einer nachhaltigen Entwicklung wäre es das Ziel, die Verwirklichungschancen für eine nachhaltige Mobilität zu erhöhen. Wenn die Menschen fähig sind sich nachhaltig zu verhalten, sollte die reale Freiheit geschaffen werden, dies auch zu tun. Da angesichts des Klimawandels und der grundlegenden ökologischen Gefährdung des Planeten Erde tatsächlich generell die Verwirklichungschancen der kommenden Generationen auf ein gutes Leben infrage gestellt werden, bleibt nichts Anderes übrig als den Pfad der nachhaltigen sozialen Entwicklung zu beschreiten. 


\section{Literatur}

Agora Verkehrswende. 2018. Öffentlicher Raum ist mehr wert: Ein Rechtsgutachten zu den Handlungsspielräumen in Kommunen. https://www.agora-verkehrswende.de/fileadmin/ Projekte/2018/OEffentlicher_Raum_ist_mehr_wert/Agora_Verkehrswende_Rechtsgutachten_oeffentlicher_Raum.pdf.

Alkire, Sabrina. 2005. Needs and Capabilities. In: Reader, S. (Hrsg.): The Philosophy of Needs. Cambridge: Cambridge University Press. S. 229-251.

Altenburg, Sven, P. Gaffron; und C. Gertz. 2009. Teilhabe zu ermöglichen bedeutet Mobilität zu ermöglichen. Diskussionspapier des Arbeitskreises Innovative Verkehrspolitik der Friedrich-Ebert-Stiftung. Bonn: Hrsg. v. Friedrich-Ebert-Stiftung (Wiso-Diskurs. Expertisen und Dokumentationen zur Wirtschafts- und Sozialpolitik). https://library.fes. de/pdf-files/wiso/06482.pdf.

Arnstein, Sherry R. 1969. A ladder of citizen participation. In: Journal of the American Institute of Planners 35 (4), 216-224.

Bartelheimer, Peter. 2007. Politik der Teilhabe. Ein soziologischer Beipackzettel. Bonn: Hrsg. v. Friedrich-Ebert-Stiftung Forum Berlin. Berlin (Fachforum: Analysen \& Kommentare, 1). https://library.fes.de/pdf-files/do/04655.pdf.

Becker, Thilo. 2016. Sozialräumliche Verteilung von verkehrsbedingtem Lärm und Luftschadstoffen am Beispiel von Berlin. Dresden: https://www.qucosa.de/fileadmin/data/ qucosa/documents/20306/Becker_Verteilung_Verkehrslaerm_Luftschadstoffe.pdf.

Bonvin, Jean-Michel. 2006. Employment and Labour Market Regulation: A Capability Approach, Berichterstattung zur sozioökonomischen Entwicklung Deutschlands Werkstattbericht Zweiter Bericht, Zwischenbericht Teil I. Göttingen: https://www.soeb. de/fileadmin/redaktion/downloads/zwischenbericht_2006_teil_1.pdf.

Brocchi, Davide. 2017. Urbane Transformation. Zum guten Leben in der eigenen Stadt. Bad Homburg: VAS.

Bundesregierung, Hrsg. 2005. Lebenslagen in Deutschland: Der 2. Armuts- und Reichtumsbericht der Bundesregierung. Berichtsband. Berlin: https://www.armuts-undreichtumsbericht.de/SharedDocs/Downloads/Berichte/lebenslagen-deutschland-zweiterarmuts-reichtumsbericht.pdf?_blob=publicationFile \&v $=3$.

Cohen, Gerald A. 2008. Rescuing Justice and Equality. Cambridge: Harvard University Press.

DESTATIS Statistische Bundesamt 2018. Zahl der Woche Preise rund ums Auto seit 2000 um $36 \%$ gestiegen, https://www.destatis.de/DE/Presse/Pressemitteilungen/Zahl-derWoche/2018/PD18_38_p002.html.

Delbosc, Alexa. 2012. 'The role of well-being in transport policy'. Transport Policy 23, 25-33.

Fischer, Frank und H. Gottweis. 2012. The argumentative turn revisited. Public policy as communicative practice. Durham, NC.

Gaffron, Philine. 2016. 'Umweltgerechtigkeit und Stadtverkehr - Leitbild, Diagnosen und Handlungsoptionen'. In Handbuch der kommunalen Verkehrsplanung: Strategien, Konzepte, Maßnahmen für eine integrierte und nachhaltige Mobilität. Hrsg. Tilman Bracher, K. Dziekan, J. Gies, F. Huber, F. Kiepe, U. Reutter, K. Saary, O. Schwedes. Berlin, Bonn: Wichmann; Economica-Verlag. 
Glasze, Georg. 2002. Wohnen hinter Zäunen - bewachte Wohnkomplexe als Herausforderung für die Stadtplanung. In Jahrbuch StadtRegion 2002. Hrsg. Norbert Gestring, H. Glasauer, C. Hannemann, W. Petrowsky und J. Pohlan. Opladen: Leske und Budrich.

Healey, Patsy. 1996. The communicative turn in planning theory and its implications for spatial strategy formation. In Environment and Planning B: Planning and Design 23 (2), 217-234.

Healey, Patsy. 2012. Performing place governance collaboratively: Planning as a communicative process. In: The argumentative turn revisited: Public policy as communicative practice. Hrsg. Frank Fischer und H. Gottweis, H. Durham NC: 58-82.

Hradil, Stefan und J. Schiener. 2001. Soziale Ungleichheit in Deutschland. Opladen: 8. Aufl. Leske + Budrich (Uni-Taschenbücher, 1809).

López, Iván, J. Ortega, und M. Pardo. 2020. Mobility Infrastructures in Cities and Climate Change. An Analysis Through the Superblocks in Barcelona. In Atmosphere 11 (4), S. 410. https://doi.org/10.3390/atmos11040410.

Lucas, Karen. 2012. „Transport and Social Exclusion: Where Are We Now?“ Transport Policy 20 (März): 105-13. https://doi.org/10.1016/j.tranpol.2012.01.013.

Lugo, Adonia E. (2018): Bicycle/race: Transportation, culture, \& resistance, Microcosm Publishing, Portland, Oregon.

Meunier, Corinne. 2006. Öffentlichkeitsbeteiligung in der Bauleitplanung: Bedeutung der Aarhus-Konvention und der ihrer Umsetzung dienenden EU-Richtlinien - Öffentlichkeitsbeteiligung in Berlin-Brandenburg im Praxistest - Arbeitshilfe für die Praxis. Dortmund: UVP spezial 20.

Neef, Max. 1991. Human Scale Development: conception, application and further reflections. New York: The Apex Press.

Nobis, Claudia und T. Kuhnimhof. 2018. Mobilität in Deutschland: MiD Ergebnisbericht, Bonn: https://www.mobilitaet-in-deutschland.de/publikationen2017.html.

Nussbaum, Martha C., H. Pauer-Studer, Herlinde und I. Utz, Ilse, Hrsg. 2014. Gerechtigkeit oder Das gute Leben. Frankfurt am Main: Dt. Erstausg., 8. Aufl. Suhrkamp.

Nussbaum, Martha C. 2000. Woman and Human Development: The Capabilities Approach. Cambridge: Cambridge University Press.

Rammert, Alexander, S. Daubitz, O. Schwedes. 2019. Entwicklung von Mobilitätsstrategien auf Basis qualitativer Daten. Internationales Verkehrswesen. 4l2019.

Rammler, Stephan und O. Schwedes. 2018. Mobilität für alle! Gedanken zur Gerechtigkeitslücke in der Mobilitätspolitik. Hrsg. Friedrich-Ebert-Stiftung Forum Berlin. Berlin: https://library.fes.de/pdf-files/dialog/14779.pdf.

Rauschmayer, Felix; I. Omann und J. Frühmann. 2011. Needs, capabilities, and quality of life. Re-focusing Sustainable Development. In Sustainable Development: Capabilities, Needs, and Well-Being. Hrsg. Rauschmayer, Felix; I. Omann und J. Frühmann London, Routledge. S. 1-24.

Rawls, John. 1971. A Theory of Justice. Cambridge, MA: Harvard University Press.

Rawls, John. 2001. Justice as Fairness: A Restatement. Cambridge, MA: Harvard University Press.

Samuels, Jane, Hrsg. 2005. Removing unfreedoms: citizens as agents of change in urban development. London: ITDG Publ. 
Scheiner, Joachim und C. Holz-Rau. 2017. 'Women's complex daily lives: A gendered look at trip chaining and activity pattern entropy in Germany'. Transportation 44(1), 117138.

Schwedes, Oliver und S. Daubitz. 2011. Hausanschluss Mobilität. Erfahrungen und Potenziale von Erreichbarkeitsplanung. Hrsg. Verbraucherzentrale Bundesverband. Berlin: https://www.vzbv.de/sites/default/files/downloads/Hausanschluss_Mobilitaet_ vzbv_2012.pdf.

Schwedes, Oliver, S. Daubitz, A. Rammert, B. Sternkopf und M. Hoor. 2018. DP1-2_ Kleiner_Begriffskanon. Berlin: https://www.ivp.tu-berlin.de/fileadmin/fg93/ Dokumente/Discussion_Paper/DP1-2_Schwedes_et_al.pdf.

Schwedes, Oliver und A. Rammert. 2020. DP 15 Was ist Integrierte Verkehrsplanung? Hintergründe und Perspektiven einer am Menschen orientierten Planung. Berlin: https://www.ivp.tu-berlin.de/fileadmin/fg93/Dokumente/Discussion_Paper/DP15_ SchwedesRammert.pdf.

Senatsverwaltung für Stadtentwicklung und Umwelt, Hrsg. 2016. Umweltgerechtigkeit im Land Berlin. Basisbericht 2016 Berlin: https://www.berlin.de/senuvk/umwelt/ umweltgerechtigkeit/download/umweltgerechtigkeit_broschuere.pdf.

Sen, Amartya. 2012. Die Idee der Gerechtigkeit. München: Dt. Taschenbuch-Verl. (dtv, 34719).

Vella-Brodrick, Dianne und J. Stanley 2013. 'The significance of transport mobility in predicting well-being', Transport Policy 29, 236-242.

Wanner, Matthias und F. Stelzer. 2019. Reallabore - Perspektiven für ein Forschungsformat im Aufwind. in brief - Wuppertaler Impulse zur Nachhaltigkeit (07/2019) https://epub. wupperinst.org/frontdoor/deliver/index/docId/7360/file/7360_Reallabore.pdf.

Wehrheim, Jan. 2002. Raumkontrolle: von sozialer Ausgrenzung zu räumlichen Ausschluss und vice versa. Widersprüche, Jg. 22 (2002), Heft 86, S. 21-37.

World Commission on Environment and Development, Hrsg. 1987. Our Common Future. Oxford: Oxford University Press. https://www.un-documents.net/wced-ocf.htm.

Wright, Michael T., Block, Martina, von Unger, Hella 2007. Stufen der Partizipation in der Gesundheitsförderung. In: Gesundheit Berlin (Hrsg.): Dokumentation 13. Bundesweiter Kongress Armut und Gesundheit, Berlin.

Wright, Michael T., M. Block, H. von Unger, H. Kilian, S. Brandes und M. Ziesemer. 2009. Erfahrung nutzen - Wissen vertiefen - Praxis verbessern: Partizipative Entwicklung der Qualitätssicherung und Evaluation in der Gesundheitsförderung bei sozial Benachteiligten. Abschlussbericht. Hrsg. Wissenschaftszentrums Berlin für Sozialforschung (WZB) und Gesundheit Berlin-Brandenburg e. V. https://docplayer. org/17340858-Erfahrung-nutzen-wissen-vertiefen-praxis-verbessern.html.

Young, Iris Marion. 2011. Justice and the Politics of Difference Princeton. 
Open Access Dieses Kapitel wird unter der Creative Commons Namensnennung 4.0 International Lizenz (http://creativecommons.org/licenses/by/4.0/deed.de) veröffentlicht, welche die Nutzung, Vervielfältigung, Bearbeitung, Verbreitung und Wiedergabe in jeglichem Medium und Format erlaubt, sofern Sie den/die ursprünglichen Autor(en) und die Quelle ordnungsgemäß nennen, einen Link zur Creative Commons Lizenz beifügen und angeben, ob Änderungen vorgenommen wurden.

Die in diesem Kapitel enthaltenen Bilder und sonstiges Drittmaterial unterliegen ebenfalls der genannten Creative Commons Lizenz, sofern sich aus der Abbildungslegende nichts anderes ergibt. Sofern das betreffende Material nicht unter der genannten Creative Commons Lizenz steht und die betreffende Handlung nicht nach gesetzlichen Vorschriften erlaubt ist, ist für die oben aufgeführten Weiterverwendungen des Materials die Einwilligung des jeweiligen Rechteinhabers einzuholen.

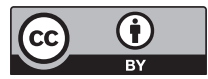

